\title{
Physical Origin and Generic Control of Magnonic Band Gaps of Dipole-Exchange Spin Waves in Width-Modulated Nanostrip Waveguides
}

\author{
Ki-Suk Lee, Dong-Soo Han, and Sang-Koog Kim* \\ Research Center for Spin Dynamics \& Spin Wave Devices and Nanospinics Laboratory, \\ Department of Materials Science and Engineering, Seoul National University, Seoul 151-744, Republic of Korea
} (Received 7 November 2008; revised manuscript received 31 January 2009; published 25 March 2009)

\begin{abstract}
We report, for the first time, on a novel planar structure of magnonic-crystal waveguides, made of a single magnetic material, in which the allowed and forbidden bands of propagating dipole-exchange spin waves can be manipulated by the periodic modulation of different widths in thin-film nanostrips. The origin of the presence of several magnonic wide band gaps and the crucial parameters for controlling those band gaps of the order of $\sim 10 \mathrm{GHz}$ are found by micromagnetic numerical and analytical calculations. This work can offer a route to the potential application to broadband spin wave filters in the gigahertz frequency range.
\end{abstract}

DOI: 10.1103/PhysRevLett.102.127202

PACS numbers: 75.40.Gb, 75.30.Ds, 75.40.Mg

The engineering of electronic band gaps in a periodic atomic structure has played a crucial role in the development of currently advanced semiconductor technologies. Reliable manipulations of the propagations of electrons in atomic-scale periodic structures as well as electromagnetic waves (photons) in submicron- or larger-scale structures are one of the long-standing fundamental issues in the field of condensed matter physics. Controlling the propagation of photons in a variety of artificially fabricated periodic structures known as photonic crystals is a good example [1]. Owing to various applications of the photonic crystal to optical nanodevices such as photonic waveguides [2] and integrated circuits [3], the photonic crystals have been given considerable attention. Meanwhile, in the areas of nanomagnetism and magnetization $(\mathbf{M})$ dynamics, the magnetic counterpart of the photonic crystals, the so-called magnonic crystal (MC), is a subject of growing interest, owing to its applications to spin wave (SW) waveguides and filters [4]. In recent years, many theoretical and experimental studies have been conducted on not only various types of MCs including one-dimensional (1D) structures, such as periodic multilayers $[5,6]$, periodic arrays of nanostrips [7], corrugated films [8,9], and comblike [10] or serial loop structures [11], but also 2D or 3D structures [12-14]. In such structures, the allowed and forbidden SW modes (called magnons) are controllable by periodic structures artificially fabricated with different magnetic material parameters [5,6,15], shapes [8-11], and exchange-bias fields [16].

Despite recent advances in fundamental understandings of those MCs as well as the wave properties of excited SW modes, few studies have focused on MC waveguides composed of simple structures for its practical application to broadband SW filters [9]. For future SW-based signal processing devices $[17,18]$, it is necessary to find micrometer-size (or smaller) MC waveguides having sim- ple planar structures, with controllable wide band gaps (of a few gigahertz) of dipole-exchange spin waves (DESWs).

In this Letter, we report, for the first time, on a new type of simple planar-patterned thin-film nanostrip waveguides in which the DESWs' magnonic bands along with their wide band gaps on the order of $10 \mathrm{GHz}$ can be manipulated by periodic modulations of different widths (of a few tens of nanometers). The physical origin of the presence of magnonic wide band gaps in such width-modulated nanostrips and the relations of the allowed DESW modes and band gaps to geometric variation of the proposed MCs were found by micromagnetic numerical and analytical calculations.

We performed micromagnetic simulations on DESW propagations in magnetic thin-film nanostrips. We used, as a model system, $10 \mathrm{~nm}$-thick Permalloy (Py) nanostrips of different widths (24 and $30 \mathrm{~nm}$ here, for example) modulating with a periodicity $P$ ranging from 12 to $42 \mathrm{~nm}$ [the light-gray area in Fig. 1(a)], which were con-

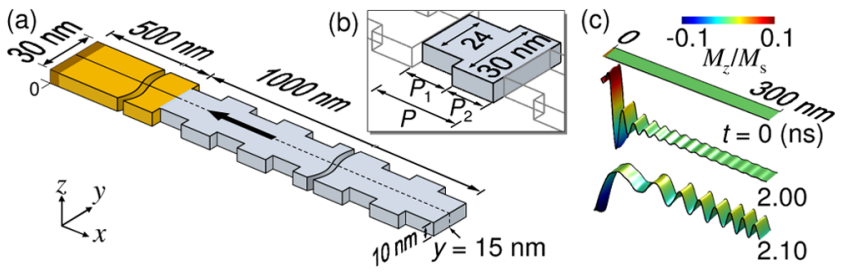

FIG. 1 (color online). (a) Geometry and dimensions of proposed nanostrip MCs with periodic modulation of different strip widths. The initial M's point in the $-x$ direction, as indicated by the black arrow. The dark-brown and yellow areas indicate the SW generation and waveguide component, respectively. (b) The unit period of $P=P_{1}+P_{2}$, where $P_{1}$ and $P_{2}$ are the segment lengths of 24 and $30 \mathrm{~nm}$ widths, respectively. (c) Temporal evolution of spatial $M_{z} / M_{s}$ distribution excited by a sinc function field with $H_{0}=1.0 \mathrm{~T}$ applied along the $y$ axis to only the dark-brown area. 
nected directly to a segment of the Py nanostrip of $10 \mathrm{~nm}$ thickness and $30 \mathrm{~nm}$ width [the yellow area in Fig. 1(a)]. The unit period of the nanostrips consists of the same Py segments with the different widths of 24 and $30 \mathrm{~nm}$ and with the corresponding lengths $P_{1}$ and $P_{2}$, respectively, as illustrated in Fig. 1(b). The OOMMF code (version 1.2a4) [19] was used to numerically calculate the dynamics of the M's of individual unit cells (size: $1.5 \times$ $1.5 \times 10 \mathrm{~nm}^{3}$ [20]) interacting through exchange and dipolar forces, which code uses the Landau-Lifshitz-Gilbert equation of motion [21]. The chosen material parameters corresponding to Py are as follows: the saturation magnetization $M_{s}=8.6 \times 10^{5} \mathrm{~A} / \mathrm{m}$, the exchange stiffness $A_{\text {ex }}=1.3 \times 10^{-11} \mathrm{~J} / \mathrm{m}$, the damping constant $\alpha=0.01$, the gyromagnetic ratio $\gamma=2.21 \times 10^{5} \mathrm{~m} / \mathrm{As}$, and with zero magnetocrystalline anisotropy. For the local homogeneous excitation and subsequent propagation of the lowestmode DESWs, along the length direction, with frequencies $f_{\mathrm{SW}}$, ranging from 0 to $100 \mathrm{GHz}$, we applied a "sine cardinal (sinc)" function $H_{y}(t)=H_{0} \frac{\sin \left[2 \pi \nu_{\mathbf{H}}\left(t-t_{0}\right)\right]}{2 \pi \nu_{\mathbf{H}}\left(t-t_{0}\right)}$, with $H_{0}=1.0 \mathrm{~T}$ and the field frequency $\nu_{\mathbf{H}}=100 \mathrm{GHz}$, only to a local area of $1.5 \times 30 \mathrm{~nm}^{2}$ indicated by the darkbrown color shown in Fig. 1(a).

The results obtained by the fast Fourier transform (FFT) of the temporal $M_{z} / M_{s}$ evolution for DESW propagations along the $x$ axis at $y=15 \mathrm{~nm}$ are plotted in Fig. 2 [18]. The frequency spectra clearly reveal the allowed and/or forbidden bands of DESWs propagating through the nanostrips: The allowed bands are indicated by the colored region, and the forbidden bands by the white region. For comparison, the fundamental mode DESWs propagating in single-width $(24$ and $30 \mathrm{~nm})$ nanostrips are shown in Figs. 2(a) and 2(b). Obviously, there is no forbidden band except for below the intrinsic potential barrier $(<14 \mathrm{GHz})$, owing to the quantization of the lowest mode of DESWs due to the geometric confinement of the nanostrip's narrow width [18,22-25]. For the different-width-modulated nanostrips, by contrast, there are several wide forbidden bands of the order of $\sim 10 \mathrm{GHz}$ [see Figs. 2(c) and 2(d)]. Moreover, the number of forbidden bands as well as the bands' position and gap width differ according to not only $P$ but

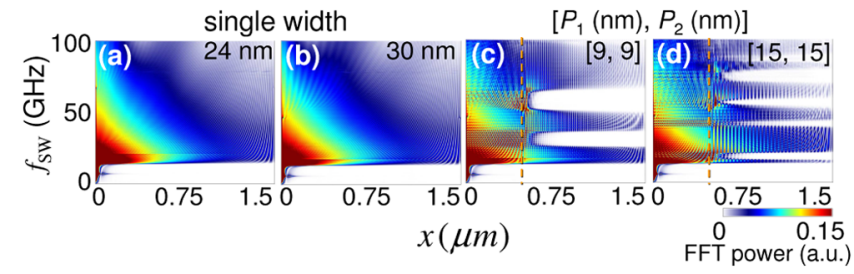

FIG. 2 (color online). Frequency spectra obtained from FFTs of $M_{z} / M_{s}$ oscillation along the $x$ axis at $y=15 \mathrm{~nm}$, for singlewidth nanostrips $(24$ and $30 \mathrm{~nm})$ and for MCs of different $\left[P_{1}\right.$ and $P_{2}$ ] values noted. The vertical dashed orange lines indicate the boundary between the single-width nanostrip waveguide [the yellow area in Fig. 1(a)] and the MC of the width-modulated nanostrip [the light-gray area in Fig. 1(a)]. also the motif (represented by $P_{1} / P$ ). More specifically, for $P=18 \mathrm{~nm}$ with $\left[P_{1}, P_{2}\right]=[9 \mathrm{~nm}, 9 \mathrm{~nm}]$, two wide band gaps (11 and $16 \mathrm{GHz}$ ) appear in the DESW modes ranging from 14 to $100 \mathrm{GHz}$, whereas, for $P=30 \mathrm{~nm}$ with $\left[P_{1}, P_{2}\right]=[15 \mathrm{~nm}, 15 \mathrm{~nm}]$, five forbidden bands with smaller gap widths $(3.8-8.6 \mathrm{GHz})$ exist (for more data, see supplementary Fig. 1 [26]).

To comprehensively understand such striking band-gap features, we plotted the dispersion curves of the DESW modes in the 24 and $30 \mathrm{~nm}$-wide nanostrips and in the nanostrip of $\left[P_{1}, P_{2}\right]=[9 \mathrm{~nm}, 9 \mathrm{~nm}][27]$ as an example. Because of the pinning of DESWs at the longer (length direction) edges of the nanostrips, there exist certain width modes having quantized $k_{y}$ values [23]. Generally, in single-width nanostrips, it is expected that the several width modes are excited [22-25], and, thus, several concave branches appear in the dispersion curves [18]. In the present simulation, however, there was a single parabolic dispersion curve, as shown in Fig. 3(a), because homogeneous DESW excitations along the width direction employed in this study led to only the lowest mode having the smallest $k_{y}$ value [see Fig. 1(c)]. Accordingly, one would expect the dispersion curves for the widthmodulated nanostrips to be folded and have band gaps at the Brillouin zone (BZ) boundaries, similar to those found typically in a $1 \mathrm{D}$ periodic system $[2,28]$. However, the dispersion curves for $\left[P_{1}, P_{2}\right]=[9 \mathrm{~nm}, 9 \mathrm{~nm}]$ show rather more complicated band features [Fig. 3(b)]: The band gaps occur not only at the $\mathrm{BZ}$ boundaries, $k_{x}=n \pi / P$ with integers $n$ (black dashed lines), but also at certain $k$ values, $k_{x}=[(2 n+1) \pi \pm 1.44] / P$ (red dotted lines). The former can be explained by a periodic translation symmetry associated with the different width modulation along the DESW propagation direction, but the latter cannot be understood by such a 1D approach.

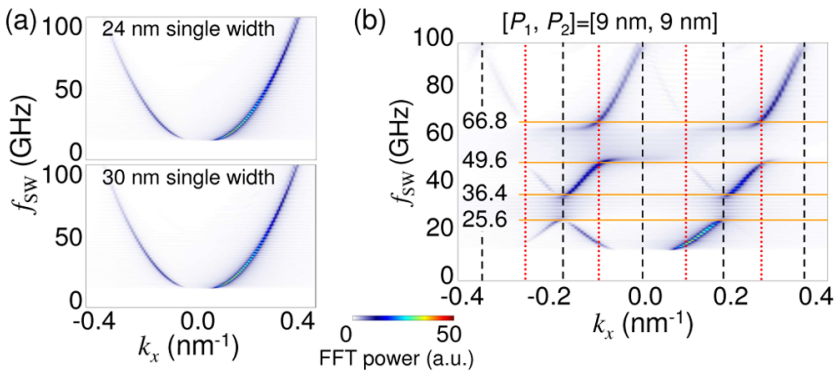

FIG. 3 (color online). (a) Dispersion curves for DESWs propagating through single-width nanostrips of 24 and $30 \mathrm{~nm}$. (b) Dispersion curves of DESWs existing in the MC of $\left[P_{1}, P_{2}\right]=[9 \mathrm{~nm}, 9 \mathrm{~nm}]$ within the nanostrip area only, from $x=501$ to $1500 \mathrm{~nm}$, obtained from FFTs of temporal $M_{z} / M_{s}$ oscillations along the $x$ axis at $y=15 \mathrm{~nm}$. The black dashed lines indicate the Brillouin zone boundaries $k=n \pi / P$, where $n=0, \pm 1, \pm 2, \ldots$, and the red dotted lines denote certain $k$ values, $k_{x}=[(2 n+1) \pi \pm 1.44] / P$ at which the forbidden band gaps occur. 
In order to quantitatively elucidate the physical origin of such different band gaps varying with different width modulation, we compared magnonic band diagrams [the thick black lines in Fig. 4(a)] obtained numerically from micromagnetic simulations for the case of $\left[P_{1}, P_{2}\right]=$ [9 nm, $9 \mathrm{~nm}$ ], combined with the analytical calculation of the band structure of a single-width $(27 \mathrm{~nm})$ nanostrip: Note that this width is just the average of the 24 and $30 \mathrm{~nm}$ widths employed in the width-modulated nanostrips. For the $27 \mathrm{~nm}$-wide nanostrip, the dispersion relation was analytically derived and expressed in terms of a quantized in-plane wave vector $\kappa_{m}^{2}=k_{x}^{2}+k_{y, m}^{2}$, with integers $m=$ $1,2,3$, etc. $[18,23]$. The $k_{x}$ and $k_{y}$ correspond to the longitudinal and transverse components of $\kappa_{m}$, respectively. The $k_{y, m}$ value can be obtained by considering the "effective" pinning [23], for example, $k_{y, m}=m \times 0.072 \mathrm{~nm}^{-1}$ for the 27 nm-wide nanostrip [29]. In Fig. 4(a), the solid red line indicates the dispersion curves of the DESW mode with $m=1$, the lowest mode excited. Owing to the periodicity of the width modulation, the dispersion curves are folded at the first BZ boundary (the dashed vertical line), as shown in Fig. 4(a), and thus these folded branches intersect with the original one at the BZ boundaries. Such a crossing of the dispersion curves indicates the "diagonal" coupling between the two identical modes having opposite propagation vectors [30]. This diagonal coupling represents interference between the initially propagating forward mode and its backward mode reflected at the BZ boundary, resulting in the standing wave pattern of DESWs with $k_{x}=$ $n \pi / P$ in the MC of $P$, and a split in the energy band (a band gap) [see the thick black lines in Fig. 4(a)] [28]. Next, Fig. 4(b) shows calculations of the spatial distributions of
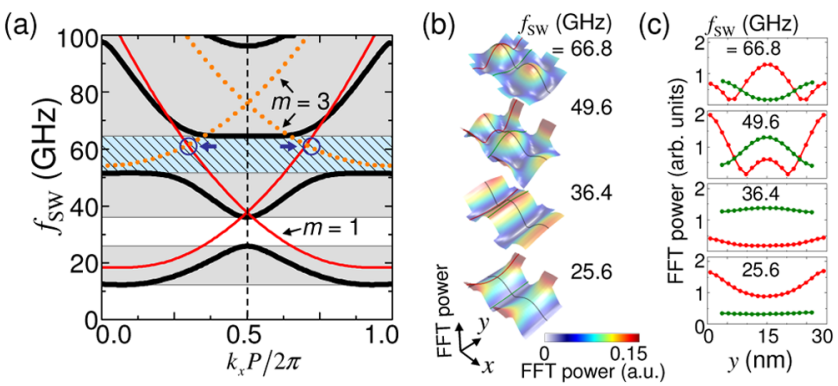

FIG. 4 (color online). (a) Comparison of magnonic band structure (black thick curves) of a nanostrip-type $\mathrm{MC}$ of $\left[P_{1}, P_{2}\right]=$ [9 $\mathrm{nm}, 9 \mathrm{~nm}$ ] obtained from micromagnetic simulations and that of a single-width nanostrip of $27 \mathrm{~nm}$ width, for two different modes of $m=1$ (solid red curves) and $m=3$ (dotted orange curves) obtained from the analytical form of Eq. (1) in Ref. [18]. (b) Perspective view of the FFT power distributions of local $M_{z} / M_{s}$ oscillations for specific frequencies for the top and bottom of allowed bands, as indicated by orange horizontal lines in the dispersion curves shown in Fig. 3(b). (c) Cross-sectional FFT power profiles of standing wave modes in the width direction ( $y$ direction). The red (green) line indicates the standing wave profile in the width direction at the center of the $30 \mathrm{~nm}$ (24 nm-) wide segment. the FFT powers of the local $M_{z} / M_{s}$ oscillations for the indicated specific frequencies selected at the top and bottom of the magnonic band for the case of $\left[P_{1}, P_{2}\right]=$ [9 $\mathrm{nm}, 9 \mathrm{~nm}$ ]. It is evident that the origin of the first band gap [31] is the diagonal coupling between the two identical but oppositely propagating lowest-mode $(m=1)$ DESWs, as explained above.

In addition to the first band gap at the BZ boundaries associated with the diagonal coupling, the dispersion branch of a higher-quantized width mode $(m=3)$, noted by the dotted orange lines in Fig. 4(a), intersects with that of the lowest mode $(m=1)$ at $k_{x}=[0.5 \pm 0.2] 2 \pi / P$ (away from the BZ boundaries) indicated by the blue circles and the arrows in Fig. 4(a). To understand these band gaps, we consider a 2D scattering of the lowestmode $(m=1)$ DESWs from the edge steps between the narrower- and wider-width strip segments [see Fig. 1(b)]. Such edge steps periodically arranged in the widthmodulated nanostrips play a crucial role as new sources for excitations of the higher width modes $(m=3,5,7, \ldots)$ [32]. In general, those DESWs scattered from the edge steps propagate in wide angles on the $x-y$ plane, so that they interfere destructively with themselves. However, for the phase-matching condition of the scattered DESWs, they can interfere constructively with themselves; in other words, other higher-width-mode $(m=3)$ DESWs being propagated in the opposite direction are excited and interact with the initial propagating lowest mode. Consequently, the interactions between the initial lowestmode $(m=1)$ and the excited higher width-mode $(m=3)$ DESWs lead to quite complicated 2D standing wave patterns of DESWs. For $f_{\mathrm{SW}}=66.8 \mathrm{GHz}$, the nodes appear in both the width and length direction [see Figs. 4(b) and 4(c)], subsequently leading to complex 2D normal modes in thin-film nanostrips of the lateral confinements [33]. This gives rise to the anticrossing of dispersion curves as well as band gaps [30] [see the thick black lines for the second and the third bands and the diagonal-line-patterned blue region between them in Fig. 4(a)].

Such strong coupling between the initially propagating mode and the newly excited higher mode and the resulting band gaps are known as the mini stop bands of electromagnetic waves in photonic crystal waveguides [2,30,34]. It is worthwhile to note that the lateral geometric confinements in the width-modulated nanostrips can also yield significant internal field inhomogeneities, as reported for a Daemon-Eschbach geometry in Refs. [24,25]. Simulation results for the dynamic $\mathbf{M}$ profiles of the normal modes between the uniform and nonuniform internal field (see supplementary Fig. 2 [26]) reveal that the dynamic M profiles in the width-modulated nanostrips could not be explained simply in terms of the inhomogeneity of the internal field. The complex modes of DESWs and associated band gaps in the width-modulated MCs are the result of the cooperative phenomena of the diffraction, reflection of the DESWs scattered at the edge steps of the widthmodulated nanostrips, and their interference with the ini- 
tially propagating lowest-mode DESWs [26]. On the basis of such novel DESW band structures, it was found that magnonic band gaps vary sensitively according to both the periodicity and the motif in width-modulated nanostrips. From an application perspective, this novel property can be implemented as an effective means of manipulating the allowed DESW modes in their propagations through such width-modulated nanostrips, a new type of SW waveguides that pass DESWs in a chosen narrow-band frequency region but filter out most DESWs having other frequencies. Moreover, these results can resolve the bottleneck of spin wave devices-the trade-off among their speed, miniaturization, and controllability by applied magnetic fields [35].

In conclusion, we found that complex DESW band structures and wide band gaps originate from the diagonal coupling between the identical lowest modes, as well as the coupling between the initially propagating lowest mode and the higher-quantized width mode newly excited through the DESWs scattering at the edge steps of different-width-modulated nanostrips. Moreover, we found that the magnonic band-gap width, the position, and the number of band gaps are controllable by the periodicity and the motif of the different width modulation.

We express our thanks to B. Hillebrands and A. Slavin for their careful reading of this manuscript. This work was supported by Creative Research Initiatives (the Research Center for Spin Dynamics and Spin Wave Devices) of MEST/KOSEF.

*Corresponding author. sangkoog@snu.ac.kr

[1] E. Yablonovitch, Phys. Rev. Lett. 58, 2059 (1987).

[2] J. D. Joannopoulos, R. D. Meade, and J. N. Winn, Photonic Crystals: Molding the Flow of Light (Princeton University, Princeton, NJ, 2008), 2nd ed.

[3] N. Engheta, Science 317, 1698 (2007).

[4] R. L. Carter et al., J. Appl. Phys. 53, 2655 (1982).

[5] D. S. Deng, X. F. Jin, and R. Tao, Phys. Rev. B 66, 104435 (2002).

[6] S. A. Nikitov, Ph. Tailhades, and C.S. Tsai, J. Magn. Magn. Mater. 236, 320 (2001).

[7] M.P. Kostylev, A. A. Stashkevich, and N. A. Sergeeva, Phys. Rev. B 69, 064408 (2004); M. Kostylev et al., Appl. Phys. Lett. 92132504 (2008).

[8] C. G. Sykes, J.D. Adam, and J.H. Collins, Appl. Phys. Lett. 29, 388 (1976); P. A. Kolodin and B. Hillebrands, J. Magn. Magn. Mater. 161, 199 (1996).

[9] A. V. Chumak et al., Appl. Phys. Lett. 93, 022508 (2008).

[10] H. Al-Wahsh et al., Phys. Rev. B 59, 8709 (1999).

[11] A. Mir et al., Phys. Rev. B 64, 224403 (2001).

[12] J. O. Vasseur et al., Phys. Rev. B 54, 1043 (1996).

[13] Yu. V. Gulyaev et al., JETP Lett. 77, 567 (2003).

[14] M. Krawczyk and H. Puszkarski, Phys. Rev. B 77, 054437 (2008).
[15] V. V. Kruglyak, and R. J. Hickena, J. Magn. Magn. Mater. 306, 191 (2006); V. V. Kruglyak et al., J. Appl. Phys. 98, 014304 (2005).

[16] C. Bayer, M. P. Kostylev, and B. Hillebrands, Appl. Phys. Lett. 88, 112504 (2006).

[17] R. Hertel, W. Wulfhekel, and J. Kirschner, Phys. Rev. Lett. 93, 257202 (2004); T. Schneider et al., Appl. Phys. Lett. 92, 022505 (2008); K.-S. Lee and S.-K. Kim, J. Appl. Phys. 104, 053909 (2008).

[18] S. Choi, K.-S. Lee, K. Y. Guslienko, and S.-K. Kim, Phys. Rev. Lett. 98, 087205 (2007).

[19] A version of the OOMMF code used is 1.2a4. See http:// math.nist.gov/oommf.

[20] Simulation results using $1.5 \times 1.5 \times 2.5 \mathrm{~nm}^{3}$ are in good agreement with those using $1.5 \times 1.5 \times 10 \mathrm{~nm}^{3}$.

[21] L. D. Landau and E. M. Lifshitz, Phys. Z. Sowjetunion 8, 153 (1935); T. L. Gilbert, Phys. Rev. 100, 1243 (1955).

[22] T. W. O'Keeffe and R. W. Patterson, J. Appl. Phys. 49, 4886 (1978); R. Arias and D. L. Mills, Phys. Rev. B 70, 094414 (2004); V.E. Demidov and S. O. Demokritov, Phys. Rev. B 77, 064406 (2008).

[23] K. Yu. Guslienko et al., Phys. Rev. B 66, 132402 (2002); K. Yu. Guslienko and A. N. Slavin, Phys. Rev. B 72, 014463 (2005).

[24] M. P. Kostylev et al., Phys. Rev. B 76, 054422 (2007).

[25] V. E. Demidov et al., Appl. Phys. Lett. 92, 232503 (2008).

[26] See EPAPS Document No. E-PRLTAO-102-023915 for two figure files and five movie files. For more information on EPAPS, see http://www.aip.org/pubservs/epaps.html.

[27] Dispersion curves were obtained from the 2D FFTs of the temporal $M_{z} / M_{s}$ oscillations along the $x$ axis $(x=$ $501-1500 \mathrm{~nm}$ ) at $y=15 \mathrm{~nm}$ (the dashed line on the nanostrip MC in Fig. 1).

[28] C. Kittel, Introduction to Solid State Physics (Wiley, New York, 1996), 7th ed.

[29] In the calculation of the $k_{y, m}$ values for the $27 \mathrm{~nm}$-width nanostrip having a homogeneous internal field distribution across the strip width, the effective pinning parameter expressed by Eq. (5) in Ref. [23] was used. The exact values of $k_{y, m}$ can be obtained using Eq. (6) in Ref. [24]. However, the approximate analytical calculation described in Ref. [23] is in good agreement with the result obtained with Eq. (6) in Ref. [24] for the single-width nanostrip of longitudinally saturated magnetizations.

[30] J.-M. Lourtioz et al., Photonic Crystals: Towards Nanoscale Photonic Devices (Springer, Berlin, 2005).

[31] The top of the first allowed band $\left(f_{\mathrm{SW}}=25.6 \mathrm{GHz}\right)$ and the bottom of the second allowed band $\left(f_{\mathrm{SW}}=36.4 \mathrm{GHz}\right)$ appear at the first BZ boundary $k_{x}=\pi / P$.

[32] Since the edge steps are symmetric with respect to the $x$ axis (mirror plane), higher-quantized width modes have a mirror symmetry in the profiles along the width axis ( $y$ axis); in other words, they have odd-number $m$ values (i.e., $m=3,5,7, \ldots$ ).

[33] M. Grimsditch et al., Phys. Rev. B 69, 174428 (2004); M. Grimsditch et al., Phys. Rev. B 70, 054409 (2004).

[34] S. Olivier et al., Phys. Rev. B 63, 113311 (2001).

[35] S. V. Vasiliev et al., J. Appl. Phys. 101, 113919 (2007). 